\title{
Role of Renal Biopsy in Evaluation of Morphological Spectrum and Pathogenesis of Lupus Nephritis
}

\author{
Archana Chirag Buch, Rupali Bavikar*, Shreya Rajesh Patel, Swapnil karnik and Jehan Ansari \\ Dr. D.Y. Patil Medical College , Hospital And Research Center , Pune (India)
}

\begin{abstract}
Introduction: Lupus nephritis is a common complication in patients with systemic lupus erythematous (SLE) characterized by deposition of antibodies in glomerular, vascular and tubulointerstitial compartments of the kidney. International society of Nephrology/ Renal Pathology Society (ISN/RPS) 2003 classification plays an important role in the diagnosis and predicting prognosis in these cases. The aim of this study was to evaluate the morphological changes in renal biopsy and to correlate them with the pathogenesis of lupus nephritis.
\end{abstract}

Methods: This was a cross sectional study of 50 renal biopsies of lupus nephritis from two tertiary care centers. Clinical data, lab findings, histopathology slides and immunofluorescence findings were retrieved and analysed by two senior pathologists. The activity and chronicity indices were calculated by each pathologist and were compared for inter observer variation. The activity index was correlated with immunofluorescence findings.

Results: The mean age of patient was 29.75 years with M:F ratio of 1:9.The frequency of each class according to ISN/RPS classification was class I (0\%),class II (20\%), class III (2\%),class IV (62\%),class V (14\%) and class VI (2\%). All class IV cases were global and active with maximum activity indices. The mean of activity and chronicity indices of pathologist A was 8.83, 2.7 and pathologist B was 9.13, 1.9. High activity showed increased intensity $(3+)$ of $\mathrm{IgG}$ and $\mathrm{C} 3$ staining on immunofluorescence.

Conclusion: We found that activity and chronicity indices calculated on renal biopsy along with immunofluorescence findings can suggest the extent of pathogenesis of lupus nephritis. This can be useful for further management and follow up.

Keywords: Lupus nephritis, SLE, Renal Biopsy,

\section{Introduction}

Lupus nephritis is a major cause of morbidity and mortality affecting $70 \%$ of patients with systemic lupus erythematosus (SLE). ${ }^{[1]}$ Lupus nephritis is manifested by proteinuria, active urinary sediments and progressive renal dysfunction. It follows relapsing, remitting pattern which may vary from patient to patient. ${ }^{[2]}$ Lupus nephritis is characterized by anti dsDNA antibodies and immune mediated injuries which lead to variety of morphological changes in glomerular, vascular and tubulointestitial compartments of kidney. These changes vary depending on deposition of different subsets of antibodies at different phases of the disease which can be demonstrated by immunofluorescence. ${ }^{[3]}$ Morphology of lupus nephritis is graded along with activity and chronicity by the modified International society of Nephrology /Renal Pathology Society (ISN/RPS) 2003 classification. This classification is useful for prognosis and therapeutic management. We aim to study the morphological spectrum including immunofluorescence findings of renal biopsies of lupus nephritis and correlate it with pathogenesis. The objectives of our study were to classify all the renal biopsies of lupus patients according to modified ISN/RPS classification, to determine activity and chronicity indices, to study the interobserver variation in calculation of these indices and to correlate morphological and immunofluorescence findings with pathogenesis.

\section{Material and Methods}

This was a cross sectional study enrolling 50 renal biopsies over a period of two years (2014-2016) diagnosed as lupus nephritis from two large tertiary medical centers in Pune (Western Maharashtra). All the patients with antinuclear antibody and anti dsDNA positive cases with adequate renal biopsy having both histopathology and immunofluorescence reports were included in the study. The patients with inadequate renal biopsy that includes absence of glomeruli and lack of tissue for immunofluorescence were excluded from the study.

Slides, blocks and relevant clinical data along with immunofluorescence findings of the renal biopsies were retrieved from the archives of department of Pathology of both the institutes. Institutional ethical committee clearance was taken.

The renal biopsy slides were studied by light microscopy using Haematoxylin and Eosin (H\&E), Periodic acid Schiff 
(PAS), Masson trichome and Jones methenamine silver stains. Renal biopsies were classified based on modified ISN/RPS classification. ${ }^{4}$ The activity and chronicity indices were calculated independently by two senior pathologists. Active lesions were considered according to presence of cellular crescents, fibrinoid necrosis, endocapillary proliferation, leucocytic infiltration, large subendothelial deposits and interstitial inflammation. ${ }^{5}$ The chronicity was graded based on glomerulosclerosis, tubular atrophy, interstitial fibrosis and fibrous crescents. ${ }^{5}$ Each activity and chronicity factor was graded on a scale of 0-3 depending on the percentage of involvement of glomeruli : 0 (absence of lesion), 1 (25\% of glomeruli involved), 2 ( lesions involving $25-50 \%$ of glomeruli), 3 (lesions involving $>50 \%$ of glomeruli). The activity factors, cellular crescents and necrosis were multiplied by factor 2 . Total was taken as activity index out of maximum of 24 . Total chronicity index was out of 12 .

The immunofluorescence for immunoglobulin $\mathrm{G}$ ( $\mathrm{IgG}$ ), immunoglobulin M (IgM), immunoglobulin A (IgA), C3, $\mathrm{C} 1 \mathrm{q}$ findings were retrieved from the data. The intensity of fluorescence was graded from 0-3.

The findings were analyzed by Microsoft Excel and statistical package Win pepi (version 11.65). Descriptive data was presented as mean and percentages and the agreement between two raters was done by interclass correlation coefficient.

\section{Results}

The mean age of patient was 29.75 years with the range of 14 to 64 years. The male to female ratio was 1:9. The prevalence of various classes of lupus nephritis is as shown in Table 1.Twenty two patients (44\%) were diagnosed as nephrotic syndrome on admission, six (12\%) were diagnosed as acute nephritic syndrome, two patients (4\%) presented as rapidly progressive nephritic syndrome according to the WHO criteria. The remaining 20 patients (40\%) showed urinary abnormalities such as microscopic hematuria and /or proteinuria. Serum creatinine levels were raised in almost all cases ranging from $1.2-8 \mathrm{mg} / \mathrm{dl}$, higher values were seen in class IV, V and VI of ISN/RPN classification.

The average number of glomeruli were 10.4 among all renal biopsies. The maximum number of cases belonged to class IV diffuse lupus nephritis. All cases of class IV were of active diffuse global proliferative type of lupus nephritis. Segmental lesions were not found. The different morphological findings of various classes are as shown in Figure 1.
The activity index was calculated on H\&E stained slides on light microscopy according to the Austin et al scoring system. ${ }^{[5]}$ We divided the wide range of activity into three groups. Mild activity when the activity index was between zero to eight, moderate activity when it was between nine to sixteen and severe activity when between seventeen to twenty four. The prevalence of activity and chronicity grading in our study is as shown in Table 2.

In our study, Class II and class V cases had low activity and chronicity indices. We had only single case of class III and VI. Class III case had mild activity (6) and chronicity (4). Class VI had moderate activity and severe chronicity. This case was probably diffuse proliferative glomerulonephritis going into advanced sclerosis lupus nephritis.

Most of our cases were diffuse proliferative lupus nephritis $(n=31)$ which revealed high activity and low chronicity index. The activity in these cases was mainly due to endocapillary proliferation (96\%), leucocytic infiltration (93.3\%), interstitial inflammation (73.3\%), subendothelial deposits $(60 \%)$, fibrinoid necrosis $(40 \%)$ and cellular crescents (11\%). The presence of wire loop and hyaline thrombi formation indicates subendothelial form of immune deposits large enough to be detected by H\&E, but better appreciated by PAS stain. These were mostly seen in class IV lupus nephritis. Various activity and chronicity are depicted in Figure 2, 3,4. The activity and chronicity indices were calculated independently by two pathologists. The results were analysed statistically as shown in Table: 3. Interobserver reliability of activity was 0.758 with $95 \%$ C.I.: 0.551 to 0.877 . Interobserver reliability of chronicity was 0.897 with $95 \%$ C.I.: 0.796 to 0.950 . Thus there was excellent correlation between the reporting of activity and chronicity index between the two pathologists.

We found that all cases of lupus nephritis were showing full house granular positivity for IgG, IgA, IgM and C3 in the glomerular basement membrane and mesangium. (Chart 1) However it was noted that the intensity of immunofluorescence varied. IgG and C3 were more intensely stained as compared to IgA and IgM. We also found IgG and C3 deposits in the tubular basement membrane. (Figure 5) Corresponding histopathology of such cases revealed tubular atrophy and interstitial inflammation. We did not have $\mathrm{Clq}$ and $\mathrm{C} 4$ results in all cases; hence were not considered for the analysis.

While analyzing activity with immunofluorescence results, we opine that renal biopsies with increased activity had high intensity of immunofluorescence staining. Whereas there was inverse relation with the chronicity. Table 4 
Table 1: Distribution of cases according to modified ISN/RNP classification.

\begin{tabular}{|l|l|c|}
\hline Class & Names & Number (\%) \\
\hline I & Minimal mesangial Lupus nephritis & $10)$ \\
\hline II & Mesangial proliferative lupus nephritis & $120 \%)$ \\
\hline III & Focal lupus nephritis & $3(2 \%)$ \\
\hline IV & Diffuse lupus nephritis & $7(62 \%)$ \\
\hline V & Membranous lupus nephritis & $7(14 \%)$ \\
\hline VI & Advanced sclerosis lupus nephritis & $1(2 \%)$ \\
\hline
\end{tabular}

Table 2: Prevalence of activity and chronicity grading in lupus nephritis

\begin{tabular}{|l|c|c|c|}
\hline Grade & Mild (I) & Moderate (II) & Severe (III) \\
\hline Activity & $32(64 \%)$ & $18(36 \%)$ & $0(0 \%)$ \\
\hline Chronicity & $43(86 \%)$ & $6(12 \%)$ & $1(2 \%)$ \\
\hline
\end{tabular}

Table 3: Inter observer variation

\begin{tabular}{|l|c|c|c|}
\hline Pathologist & A (Mean +/- SD) & B (Mean +l- SD) & 95\% C. I. \\
\hline Activity index & $8.83+/-2.73$ & $9.13+/-3.20$ & -0.47 to 1.07. \\
\hline Chronicity index & $2.7+/-2.58$ & $1.97+/-2.72$ & 0.28 to1.18 \\
\hline
\end{tabular}

Table 4: Association of activity and chronicity grading with intensity of IgG.

\begin{tabular}{|l|c|c|c|c|}
\hline & No. of cases & $\mathbf{1 + N}(\%)$ & 2+ N (\%) & 3+ N (\%) \\
\hline Mild activity (0-8) & 32 & $7(21)$ & $18(56)$ & $7(21.8)$ \\
\hline Moderate activity (9-16) & 18 & $3(16.7)$ & $5(27.3)$ & $10(55)$ \\
\hline Mild chronicity (0-4) & 43 & $5(11.7)$ & $22(51.1)$ & $16(37.2)$ \\
\hline Moderate chronicity (5-8) & 6 & $1(16.7)$ & $3(50)$ & $2(33.3)$ \\
\hline Severe chronicity (9-12) & 1 & $0(0)$ & $1(100)$ & $0(0)$ \\
\hline
\end{tabular}

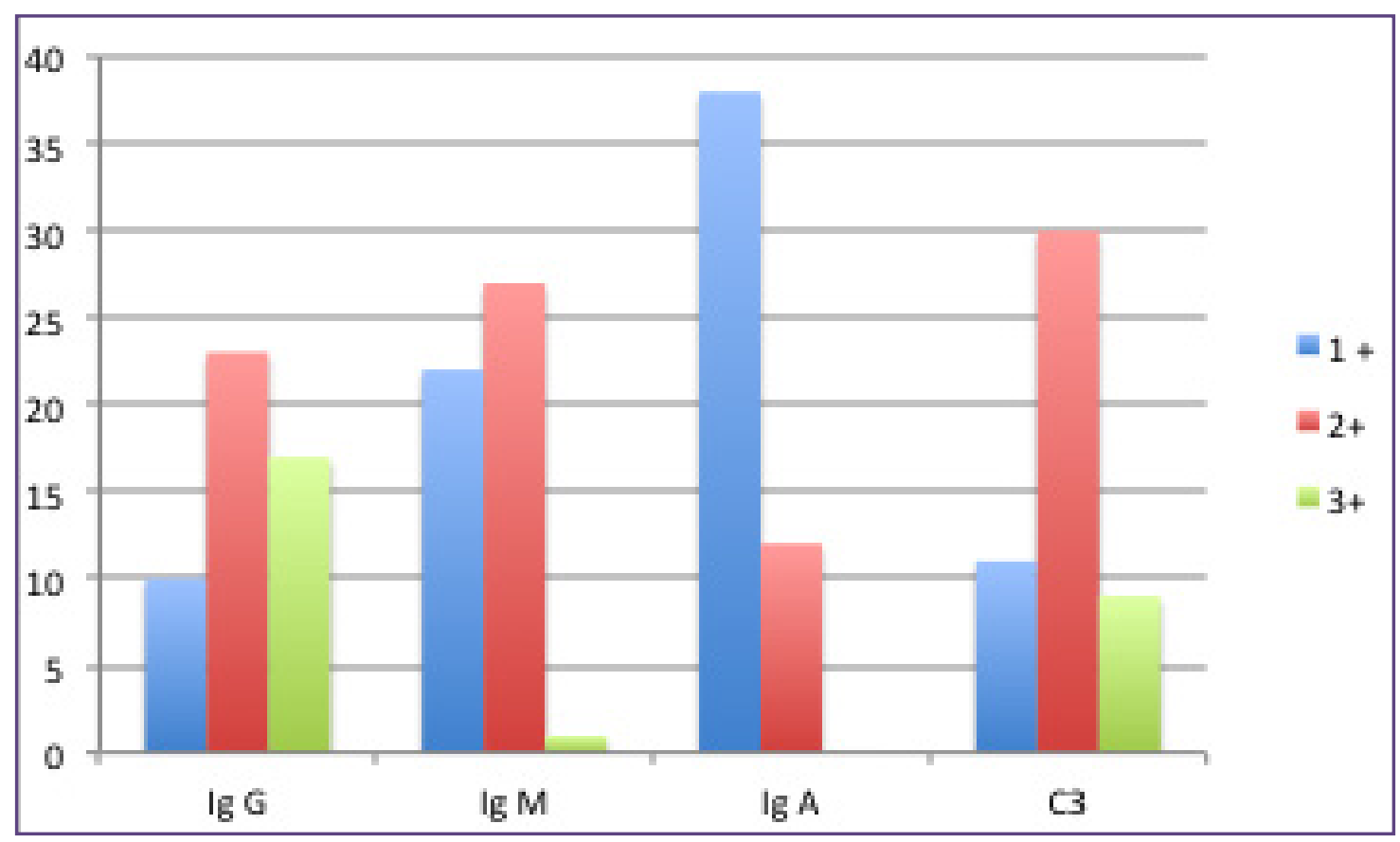

Chart 1: Correlation of severity of immunofluorescence with different antibodies. 


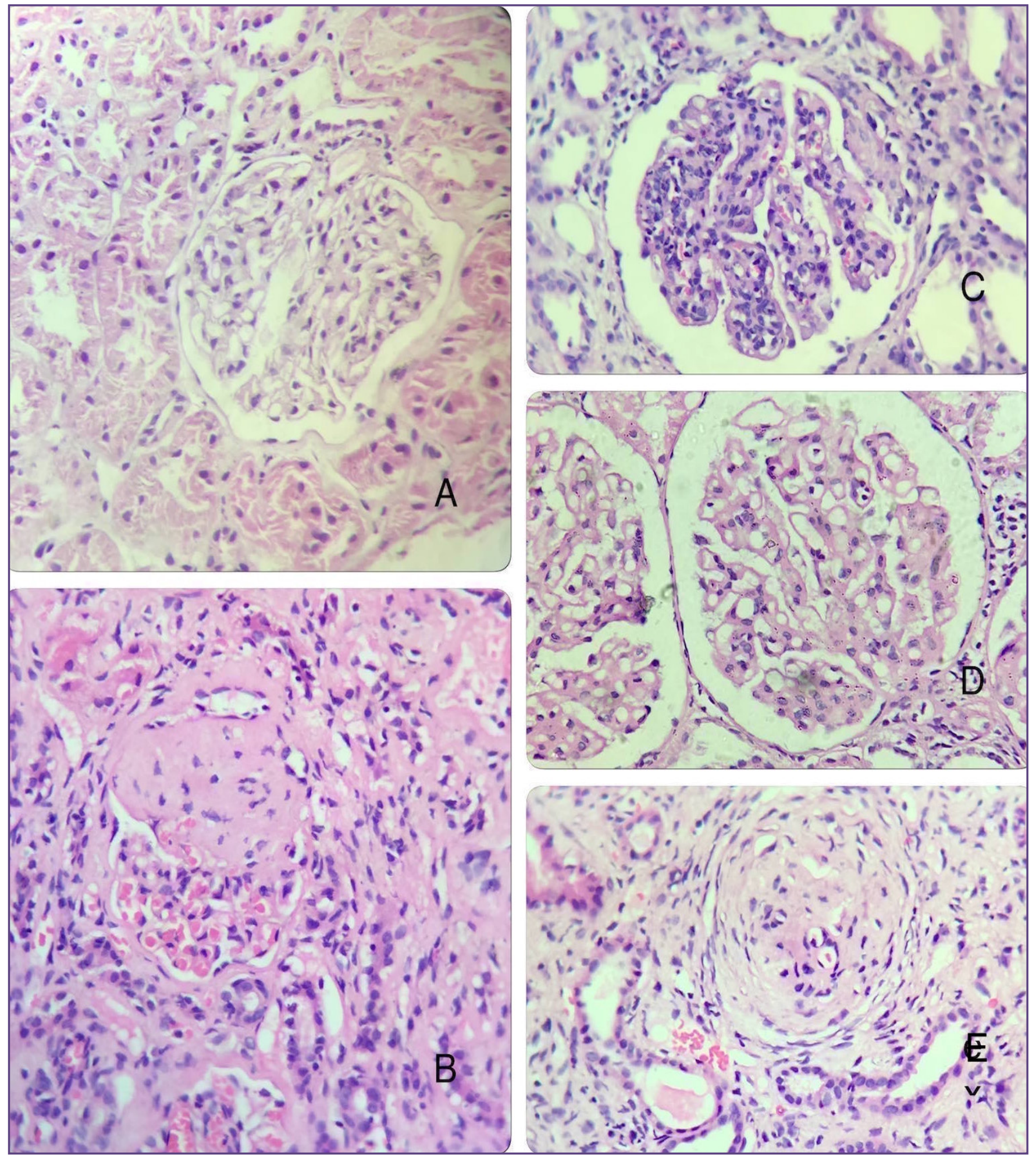

Fig. 1: A: Mesangial proliferative LN B: Focal segmental LN C : Diffuse LN D: Membranous LN E: Advance Sclerosis LN ( H\&E,X400). 


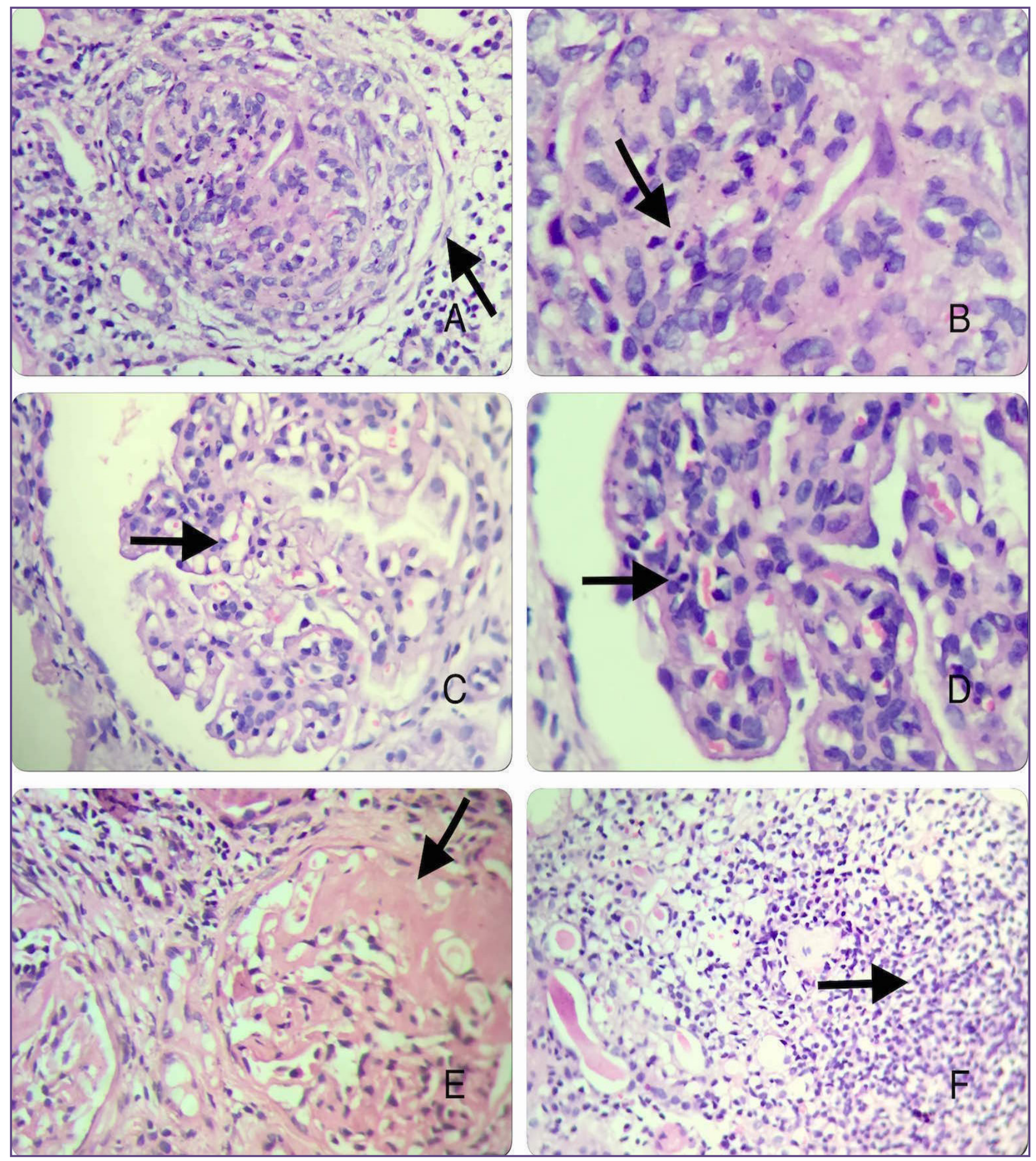

Fig. 2: A: Cellular crescents, B: fibrinoid necrosis, C: endocapillary proliferation, D: leucocytic infiltration, E: large subendothelial deposits F: interstitial inflammation. ( H\& E, X400). 


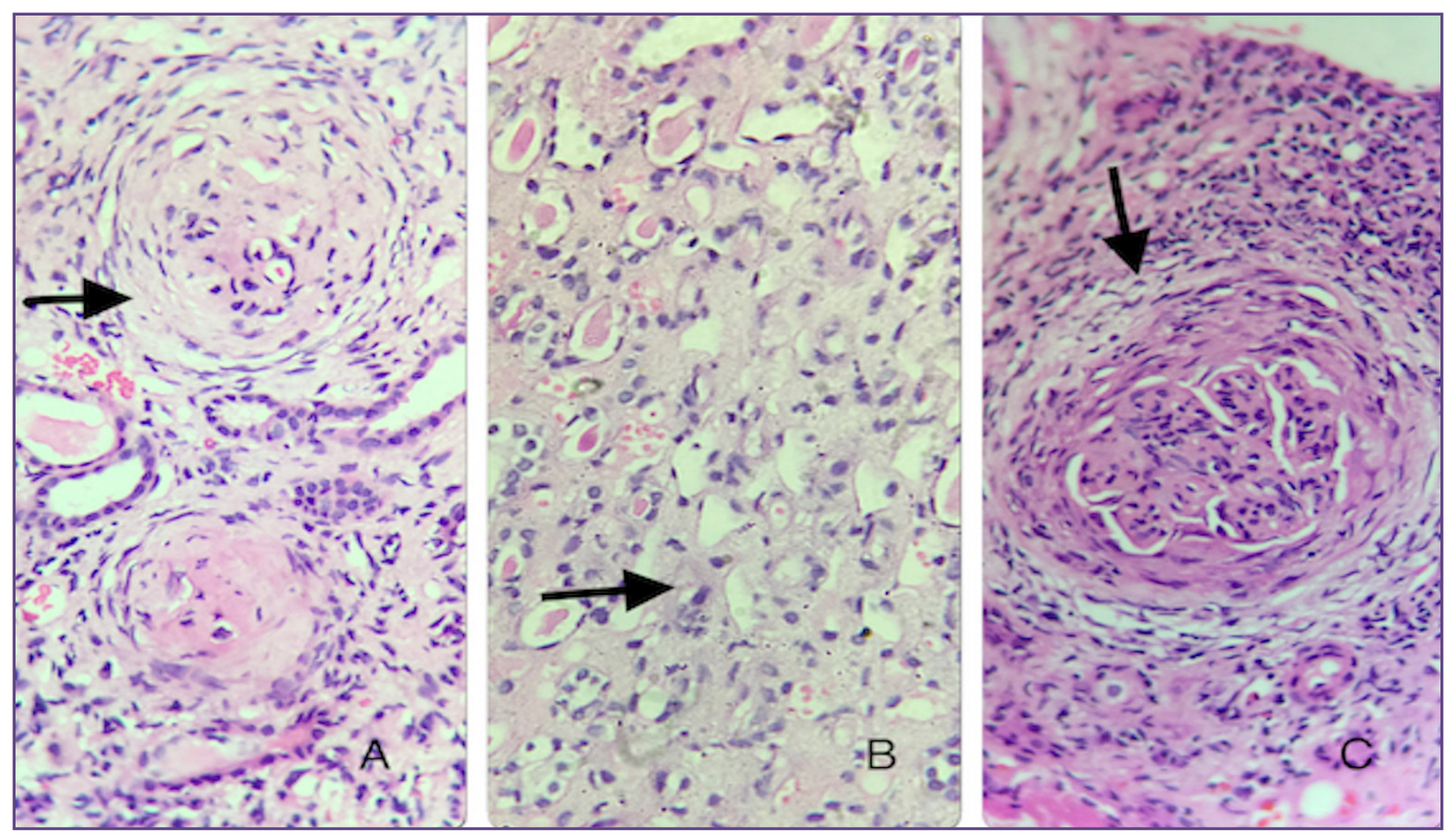

Fig. 3: A: Glomerulosclerosis B: Tubular atrophy and interstitial fibrosis C: Fibrous crescents. ( H\& E, X400).

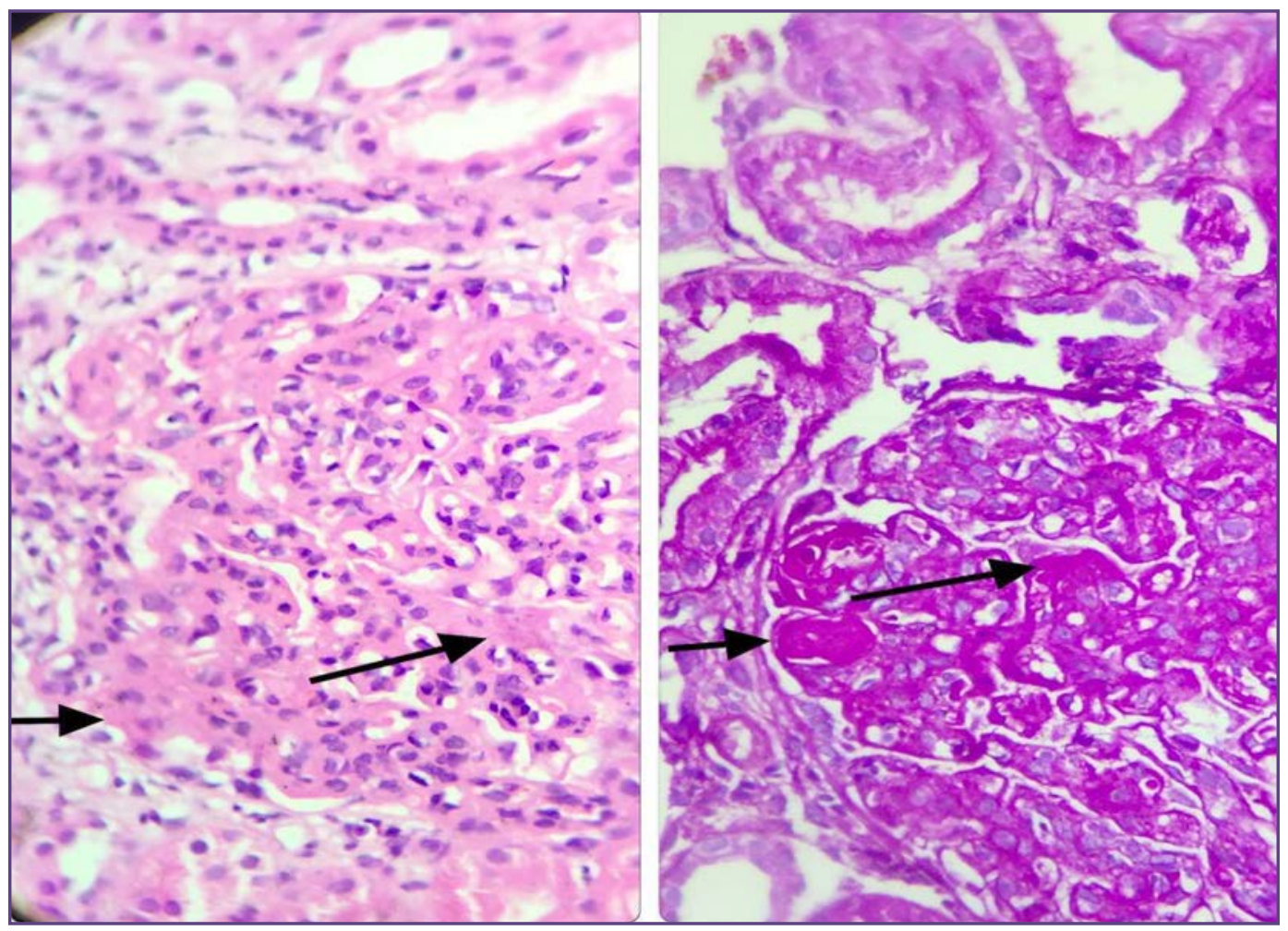

Fig. 4: A. Photomicrograph of hyaline thrombi (short arrow) ( H \& E, x400) B. Photomicrograph of hyaline thrombi ( short arrow), Wire loop lesions (long arrow) ( PAS, x400). 


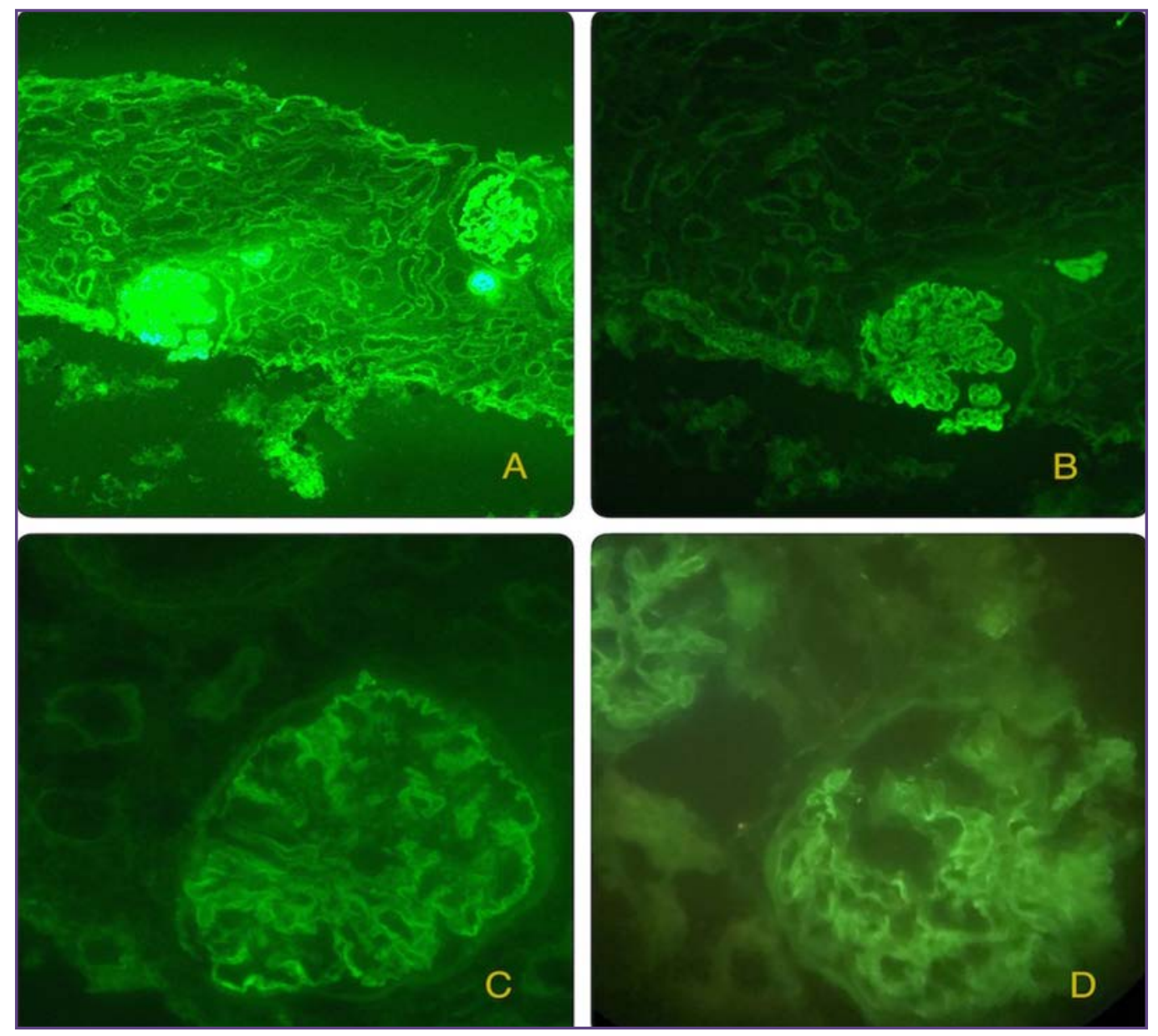

Fig. 5: Lupus nephritis Immunofluorescence A: IgG (3+) B; C 3 ( 3+); C: IgM ( 2+); IgA ( 1+).

\section{Discussion}

Systemic lupus erythematosus is a multisystem autoimmune disease characterized by development of antibodies towards chromatin material, particularly to dsDNA. There are several other antibodies such as anti nucleosome, antiRo, anti-Smith, anti-C1q, anti-alpha actinin, anti-annexin II and anti ribosomal P protein. ${ }^{[6,7]}$ These antibodies bind to various kidney structures like glomerular basement membrane, mesangial cells, glomerular epithelial cells, glomerular endothelial cell, podocytes and tubular basement membrane. ${ }^{[6,7]}$ These antibodies have pathogenic potential causing lupus nephritis.

Anti-dsDNA antibodies, actually forms a part of natural antibodies of IgM type. In lupus nephritis, these antibodies change to IgG type that increases the pathogenic potential. Anti-dsDNA antibodies are found in $70-96 \%$ of patients with lupus nephritis compared to $0.5 \%$ in patients with non lupus autoimmune disease or in healthy individual. [6]. Pathogenicity in the lupus nephritis is due to the passive entrapment of circulating dsDNA/anti-dsDNA immune complexes in the glomeruli. Deposition of immunoglobulins and activation of complement within the mesangium is a cardinal feature in lupus nephritis. Complement activation plays an important role in the pathogenesis. ${ }^{8}$ This leads to increased mesangial proliferation, apoptosis, activation of PKC and MAPK signaling pathways and increase in synthesis of proinflammatory and profibrotic mediators and cytokines. These ultimately contribute to progressive inflammatory and fibrotic process leading to various morphologic changes. The newer ISN/RPS classification grades these pathogenetic response in the form of activity and chronicity with important modifications concerning qualitative and/or quantitative differences between various subclasses. ${ }^{[9]}$

We found maximum number of female cases with mean age of 29.75 years. This was in concordance with study done by Gomma W. et al. ${ }^{[9]}$ However Nezhad ST et al had lower mean age of patients with lupus nephritis. This may be probably due to his lower sample size. Our clinical presentations of the cases were comparable with many international and national reports of lupus nephritis. ${ }^{[10-12]}$ 
The findings regarding the distribution of cases in classes are similar to reports from different region continents. We found that class IV diffuse proliferative lupus nephritis is the most common pathological lesion in our study. This finding is similar to various previous studies. ${ }^{[11-19]}$ However some studies from Arab countries showed higher incidence of Class III. ${ }^{[20,21]}$ Different ethnic group and varied sample size may be the explanation for this difference. Global active lesions were maximum in our study which is similar to findings of others. ${ }^{[12,22]}$

We had no case of Class I nephritis. Literature search also revealed no or less cases of class I nephritis. ${ }^{[9]}$ This may be explained by the fact that electron microscopy is mandatory to diagnose this lesion, as on light microscopy, all glomeruli appear normal. ${ }^{[19]}$ Renal biopsy is also performed in the patients in late stage, in most of the cases when renal functions are deranged. This also suggests that renal biopsy should be performed prior to appearance of renal symptoms in SLE patients for early detection of evidence of lupus nephritis.

The activity and chronicity index were graded as mild, moderate and severe. These reports are higher as compared with those reported by Hiramatsu et al ${ }^{[23]}$ The description of class III and IV depends on the activity and chronicity on the proportion of glomeruli with active and chronic lesions. Austin et al devised a semiquantitative scoring system for activity and chronicity by grading and adding different morphologic components in a given biopsy as a guide to treatment and prognosis. ${ }^{[12]}$ Activity index can be a significant risk factor for secondary outcome like end stage renal failure leading to death. Activity index is related to circulating gamma interferon levels thereby reflecting immunological disease activities in patients with SLE. Activity index can also be a useful guide for response to the treatment. ${ }^{5}$

We identified wire loop and hyaline thrombi formation, which indicated sub endothelial form of immune deposits large enough to be detected by $\mathrm{H} \& \mathrm{E}$, mostly in diffuse proliferative glomerulonephritis. It is believed that presence of wire loop in LM study is correlated with massive sub endothelial deposition on electron microscopy. ${ }^{[24]}$

We believe that activity index might be reflecting the severity of the immunologic disease activities in the patients with lupus nephritis. Hence activity index can be a useful clinicopathologic guide for management of lupus nephritis.

We found that mean activity was maximum in Class IV lesions and least in class V. When graded into mild, moderate and severe, maximum biopsies (64\%) showed mild activity and others (36\%) showed moderate activity. We did not have follow up data; which was one of the major limitation of our study.

Chronicity index is a measure of sclerosed glomeruli, fibrous crescents, interstitial fibrosis and tubular atrophy. Tubular atrophy and interstitial fibrosis are the main parameters related to renal function and responsiveness to therapy ${ }^{[25]}$ Variable degree of tubulointerstitial inflammation and fibrosis are found in all forms of chronic progressive renal diseases. The severity of tubulointerstitial changes inversely correlates with renal prognosis. We also found immune aggregates along the renal tubular basement membrane in few cases. It is stressed that the persistence of interstitial inflammation after therapy may predict the renal failure. Chronicity index is a better index to determine or predict the prognosis of the patients.

Grcevska L et al had a study with follow up data of 10 years which revealed that probability of renal failure 10 years after the diagnosis of lupus nephritis was higher when activity was $>11$ and chronicity of $>3$. There was no probability of renal failure in $90 \%$ in case of zero chronicity. When Chronicity was $>/=1$, there was $50 \%$ chance of renal failure. ${ }^{[26]}$ Our study revealed 21 ( 42\%) cases with zero chronicity, 22 (44\%) with 1-3 and 7 (14\%) cases were $>3$ chronicity.

Activity and chronicity indices are the major morphological parameters deciding the prognosis of the patient, it is essential to have least subjective variation. The newer classification has reduced the subjective variation by quantifying the grading criteria. We still studied the interobserver variation in identifying activity and chronicity indices by two senior nephropathologists. Interobserver variation in our study was statistically not significant. This was due to correct semiquantitative measurement for grading the activity and chronicity indices into 0-3 scale. Furthermore we found that grouping the indices in mild, moderate and severe grading almost nullifies the interobserver variation. ${ }^{[5]}$

We found that the intensity of IgG was maximum, followed by $\mathrm{C} 3$ in cases of lupus nephritis. This confirmed that the pathogenicity is mainly because of anti ds/DNA, which is IgG type of antibody and the complement activation. These findings were also in concordance with Lai $\mathrm{F}$ et al who revealed that $\mathrm{IgG}$ immunofluorescence was predominant among other immunoglobulins which were associated with C1, C4 and C3 components. ${ }^{[27]} \mathrm{We}$ have attempted to correlate the activity index with the intensity of immunofluorescence findings. We found that $55 \%$ of biopsies with moderate activity had high intensity of staining (3+) with IgG. $27.7 \%$ had intensity $2+$, and $16.6 \%$ had intensity $1+$. Whereas mild activity index cases 
revealed maximum of 2+ intensity of IgG Staining. Similar findings were also noted with intensity of $\mathrm{C} 3$ staining. Intensity of IgA, IgM were not found very significant with the activity index. Ferluga et al., showed statistically significant association between increasing mean values of the activity index and glomerular deposit distribution patterns.$^{28}$ However study done by Nossent showed no statistical significant correlation between activity index and any of the immunofluorescence intensity (all $\mathrm{P}$ values $>0.3$ ), while CI showed a negative correlation with IgA deposits only ${ }^{[29]}$

The limitation of the study is lack of electron microscopic findings of the renal biopsies due to unavailability of electron microscope facility and unaffordability. However, many studies have agreed to the fact that lack of electron microscopic findings should not prevent the skilled pathologist from rendering a diagnosis of lupus nephritis using a combination of complete light microscopy and immunofluorescence studies. ${ }^{[18]}$ We also insist on the fact that new classification of lupus nephritis with determination of activity and chronicity of the lesions, clinical data and immunofluorescence findings can help in understanding the pathogenesis and thereby helping in management and prognosis of the patient.

\section{Conclusion}

We retrospectively analyzed 50 subjects with light microscopy and immunofluorescence findings proven lupus nephritis. We found maximum cases of diffuse proliferative glomerulonephritis ( Class IV) . The activity index co related well with the intensity of immunofluorescence findings. High intensity of $\mathrm{IgG}$ and complement $\mathrm{C} 3$ confirmed the fact that pathogenicity of lupus nephritis is mainly due to anti ds DNA and complement activation which induces proinflammatory and profibrotic pathways. These can be measured by activity and chronicity index on light microscopy. Thus not only clinicopathological correlation, but detailed light microscopy and immunofluorescence findings play a very important role in judging the immunological progression of the disease; which ultimately helps in deciding the treatment and prognosis.

\section{References}

1. Cameron JS. Lupus nephritis. JASN.1999;10(2):413-24.

2. Sidiropoulos PI, Kritikos HD, Boumpas DT. Lupus nephritis flares .Lupus 2005;14(1):49-52

3. Hahn BH. Antibodies to DNA. NEJM 1998;338(19):13591368.

4. Weening JJ, D'agati D, Schwartz M, et al: The classification of glomerulonephritis in systemic lupus erythematosus revisited. Kidney Int 2004 ; 65:521-530,
5. Austin HA, Muenz LR, Joyce KM, et al: Diffuse proliferative lupus nephritis: Identification of specific pathologic features affecting renal outcome. Kidney Int 1984;25:689-95

6. Rahman A, Isenberg DA. Systemic lupus erythematosus. NEJM. 2008;358(9):929-39.

7. Mannik M, Merrill CE, Stamps LD, Wener MH. Multiple autoantibodies form the glomerular immune deposits in patients with systemic lupus erythematosus. $J$ of Rheumatology. 2003;30(7):1495-504.

8. Zhou W, Marsh JE, Sacks SH. Intrarenal synthesis of complement. Kidney International. 2001;59(4):1227-235.

9. Gomaa W, Bahlas S , HabhabW, MustaqM etal .Clinicopathological characteristic of lupus nephritis in western region of Saudi Arabia:An experience from two tertiary medical centers.Journal of Microscopy and Ultrastructure 2014;2:12-19

10. Qari.f Clinical pattern of systemic lupus erthematosus in Western Saudi Arabia ,Saudi Med J 2002;23:1247-250.

11. Heller T, AhmedM., SiddiqqiA., WallrauchC., BahlasS, Systemic lupus erythematosus in Saudi Arabia: morbidity and mortality in a multiethnic population Lupus, 2007;16:908-14

12. YokoyamaH., WadaT., HaraA., YamahanaJ., NakayaI., KobayashiM., et al. The outcome and a new ISN/RPS 2003 classification of lupus nephritis in JapaneseKidney Int 2004;66:2382-88.

13. JallouliM., FriguiM., HmidaM., MarzoukS., KaddourN., BahloulZ.Clinical and immunological manifestations of systemic lupus erythematosus: study on 146 south Tunisian patients. Saudi J Kidney Dis Transpl 2008;19:1001-8

14. Arfaj A., Khalil N., Al SalehS. Lupus nephritis among 624 cases of systemic lupus erythematosus in Riyadh, Saudi Arabia Rheumatol Int 2009;29: 1057-1067.

15. Huong D., Papo T., Beaufils H., Wechsler B., Bletry O., Baumelou A., et al. Renal involvement in systemic lupus erythematosus. A study of 180 patients from a single center Medicine (Baltimore)1999;78:148-66

16. Neumann K., Wallace D., Azen C., Nessim S., Fichman M., Metzger A., et al. Lupus in the 1980: III. Influence of clinical variables, biopsy, and treatment on the outcome in 150 patients with lupus nephritis seen at a single center Semin Arthritis Rheum1995;25:47-55

17. Seedat Y., Parag K., Ramsaroop R. Systemic lupus erythematosus and renal involvement. A South African experience Nephron1994;66:426-30

18. Shayakul C., Ong-aj-yooth L., ChirawongP., NimmannitS., ParichatikanondP., LaohapandT., et al. Lupus nephritis in Thailand: clinicopathologic findings and outcome in 569 patients Am J Kidney Dis 1995;26:300-307

19. NezhadS., SepaskhahR. Correlation of clinical and pathological findings in patients with lupus nephritis: a fiveyear experience in Iran Saudi J Kidney Dis Transpl 2008 ;19:32-40 
20. Al AttiaH., Al AhmedY., Chandani A. Serological markers in Arabs with lupus nephritis Lupus 1998;7: 198-201

21. Al-JarallahK., Al-AwadiA., SiddiquiH., Al-SalimI., ShehabI., Umamaheswaran I. , et al. Systemic lupus erythematosus in Kuwait-hospital based study. Lupus 1998;7:434-8

22. LuF. Yu, TanY., WuL., ZhuN., LiuG., ZhaoM.. Class IV-G and IV-S lupus nephritis in Chinese patients: a large cohort study from a single center Lupus 2009;18:1073-10

23. HiramatsuN., KuroiwaT., IkeuchiT., MaeshimaA., KanekoY., HiromuraK., et al. Revised classification of lupus nephritis is valuable in predicting renal outcome with an indication of the proportion of glomeruli affected by chronic lesions. Rheumatology (oxford) 2008;47:702-7

24. Guillermo AH. The valve of electron microscopy in the diagnosis and clinical management of lupus nephritis. Ultrastuct Pathol 1999;23:63-77.
25. HowieA, Turhan N.,Adu D. Powerful morphometricindicator of prognosis in lupus nephritis. QJM. 2003;96(6):411-20.

26. Grcevska1 .L, Petrusevska2 .G, Dzikova. S, Polenakovic .M. Histopathological index of Activity and Chronicity :predictors of renal outcome in lupus nephritis .BANTAO journal 2003;1(2):138

27. Lai .F, Li .P, Choi .P, K. To, Wang .A, Leung .C, et al. Histological appraisal of lupus nephritis Hong Kong J Nephrol 2000;2:13-18

28. Ferluga D, Jerse M, Vizjak A, Rozman B, Kos-Golja M, Bren AF. Correlation among WHO classes, histomorphologic patterns of glomerulonephritis and glomerular immune deposits in SLE. Wien Klin Wochenschr.2000;112 :692-701.

29. Nossent H, Berden J, Swaak T. Renal immunofluorescence and the prediction of renal outcome in patients with proliferative lupus nephritis. Lupus 2000;9(7):504-10.

*Corresponding author:

Dr Rupali Bavikar, Dr. D.Y.Patil Medical College , Hospital And Research Center , Pune (India)

Phone: 02027805000

Email: rupalibavikar@yahoo.co.uk

Date of Submission : 21.06.2017

Date of Acceptance : 05.09.2017

Financial or other Competing Interests: None.
Date of Publication : 21.12.2017 